\title{
An Ontological Approach for Semantic Modeling of Curriculum and Syllabus in Higher Education
}

\author{
Hyunsook Chung and Jeongmin Kim
}

\begin{abstract}
According to the development of new technologies of the Web, there are many researches incorporating web technologies into e-learning environment. Especially, ontology engineering has been applied to invent the semantic models of various learning entities. We have focused on the design of the integrated learning ontology conceptualizing multilevel knowledge structures, such as curriculum, syllabus, learning subject, and materials. In this paper, we present the designed curriculum ontology and syllabus ontology in detail. In addition, we propose a syllabus integration and classification method based on the definition of the semantic model of the syllabus. This approach has the contribution in terms of adaptive concept sequencing and syllabus sharing.
\end{abstract}

Index Terms-Adaptive learning path, curriculum, learning ontology, syllabus.

\section{INTRODUCTION}

As the rapid progress of the Internet and Web technologies, e-learning technologies also have been improved by applying these new web technologies for the last decades. Similar to the evolution of Web technology, the evolution of e-learning technology can be categorized into e-learning 1.0, e-learning 2.0, and e-learning 3.0 [1]. In e-learning 1.0, learning content created, stored and distributed on the Web for viewing the content online. E-learning 1.0 made learning objects available online to support easy and convenient access.

E-learning 2.0 incorporates the capability that students can access learning material passively as well as share their opinions by writing notes. E-learning 3.0 is characterized with the both of collaborative and intelligent [2]. In e-learning 3.0, students can have self-organized or personalized learning based on the application of the Semantic Web technology.

Recently, lots of e-learning related research has been trying to develop the intelligent e-learning system by incorporating ontological engineering. The researches applying ontology technology to education field are classified into curriculum or syllabus ontology creation [3], [4], ontology-based learning object organization, and ontology-based learning content retrieval. The studies for education ontology creations include curriculum ontology creation [5] and personal subject ontology creation [6].

Mizoguchi [7], [8] proposed an ontology-based solution to

Manuscript received October 10, 2014; revised December 5, 2014. This work was supported by the Korea Research Foundation Grant funded by the Korean Government (No. 2010-0006521).

Hyunsook Chung is with the Department of Computer Engineering, Chosun University, Gwangju, 501-759 South Korea (e-mail: hsch@chosun.ac.kr).

Jeongmin Kim is with the Department of Computer Engineering, Daejin University, Pocheon, 471-711, South Korea (e-mail: jmkim@daejin.ac.kr). solve several problems caused by intelligent instructional systems. There are other works to define the metadata of learning objects and learning paths based on ontology engineering technology [9], [10]. These works concentrated on the management of learning objects and materials and performance enhancement of instructional systems. Furthermore, the ontology technology can be used to make the knowledge structure, which improves the interaction among teachers and students and enables spontaneous learning of students, of teaching contents and learning materials for students based on semantic information.

Curriculum sequencing denotes the organization of learning units, i.e. courses, in an appropriate order for that students can learn their subject areas and learning tasks [3]. In e-learning filed the previous studies have proposed the approaches to construct adaptive learning paths for suitable for students individually [11], [12]. However, they are concerned only courses as learning units rather than more detailed learning concepts, which are described in learning materials or lectures, to be ordered with curriculum sequencing.

The syllabus is created by an instructor for students in order to introduce the teaching course and provide useful information and learning materials. Most students may use the course syllabus to recognize course purpose, policies, assignments, tests, out-comes, and so on. The course syllabus is an important entity as a skeleton of course, but most syllabuses only organize general information about the course like title, description, instructor, grading policy, textbook, schedule, and so on. To enable syllabus-based intelligent services, the textual unstructured syllabus should be transformed into the well-designed semantic model.

Our ongoing e-learning project has a goal to develop a learning ontology model which integrates different kinds of learning entities like curriculum, syllabus, learning subjects, and learning materials based on a layered structure. In addition, we have been developing ontology-based services which are curriculum aligning, syllabus classification, semantic retrieval of syllabuses and learning concepts, and adaptive learning path recommendation. In this paper, we present our designed learning ontology model, especially curriculum ontology and syllabus ontology in detail. In addition, we describe the integration and classification methods of the heterogeneous structured syllabuses based on the learning ontology.

\section{THE INTEGRATED LEARNING ONTOLOGY}

We consider a curriculum as the top-level information of educational data entities of a university. Generally, a curriculum is composed of a list of courses which are 
scheduled for education period. A course can be defined as a set of syllabuses, teaching and learning activities, lecture materials, assessment, and so on. A syllabus, which is a blueprint of a course, includes various important information describing instructor, learning objective, assignment, policy, teaching schedule, and so on.

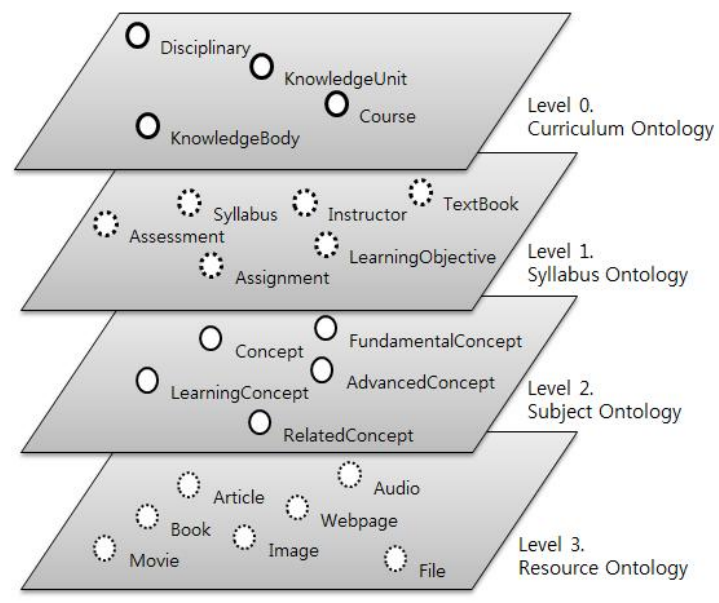

Fig. 1. Four-layered integrated learning ontology.

A weekly teaching schedule represented in a syllabus contains the core topics that students should learn in a semester. Each topic can be conceptualized with a series of learning concepts and relationships between them, which has specific learning materials and objects, such as paragraphs of a textbook, multimedia items, web pages, and so on. All above mentioned educational information should be integrated and interlinked to provide the intelligence services on e-learning environment. Fig. 1 shows the four-layered learning ontologies, which are composed of Curriculum Ontology, Syllabus Ontology, Subject Ontology, and Resource Ontology.

\section{Curriculum OnTOlogy Design}

Current most curriculum management systems of universities provide only a list of open courses and credit information about them for each department. The goal of our curriculum ontology is in the provision of a complete representation of the knowledge areas of disciplines for educators, curriculum designers, education jurisdictions, and students. Thus, the curriculum ontology can be used to support curriculum development through curriculum matching, interlinking, and classification between relevant disciplines. In addition, it covers a set of syllabus ontologies, linked to course classes defined in the curriculum ontology.

TABLE I: THE ClasSES OF CURRICULUM ONTOLOGY

\begin{tabular}{lllll}
\hline \hline Class & Label & Superclass & SubclassOf & Definition \\
\hline Discipline & Discipline & - & BodyOfKnowledge & Field of study \\
BodyOfKnowledge & Body of Knowledge & Discipline & KnowledgeArea & Main knowledge body of the discipline \\
KnowledgeArea & Knowledge Area & BodyOfKnowledge & UnitOfKnowledge & Subjects of a body of knowledge \\
UnitOfKnowledge & Unit of Knowledge & KnowledgeArea & Course & Category of a knowledge area \\
Course & Course & UnitOfKnowledge & ElectiveCourse, ... & Course \\
Competency & Competency & - & - & Learning competency \\
College & College & University & Department & College of a university \\
Department & Department & College & - & Department of a college \\
\hline \hline
\end{tabular}

In order to design the curriculum ontology, we referenced Computing Curricula 2013 [13], [14], which is an international curricular guidelines for undergraduate programs in computing related disciplines created by ACM and IEEE-Computer Society. As shown in Table I, classes defined in the curriculum ontology represent not only the existing curriculum structure, but also knowledge structure defined in CC2013. Table I describes a partial list of classes defined in the curriculum ontology.

Fig. 2 represents some relationships of the curriculum ontology established between classes, which are including hasBodyOfKnowledge, hasKnowledgeArea, hasCourse, hasMajorCourse, hasElectiveCourse, and so on. For example, a specific course titled as "Understanding Java Programming" has a subsumption relation with an instance of UnitOfKnowledge class titled as "Java Programming".

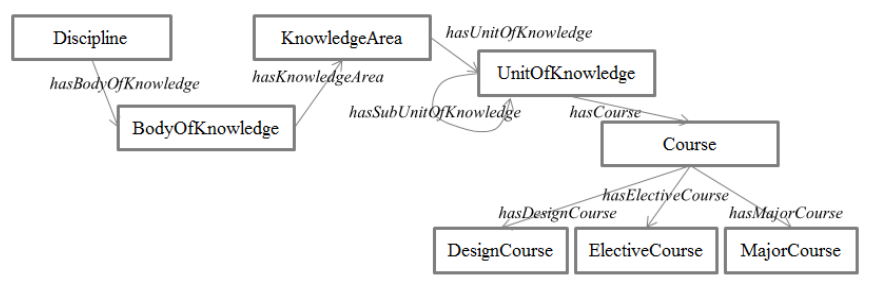

Fig. 2. Relationships between classes of the curriculum ontology.
The curriculum ontology in which all relevant concepts and relationships between the concepts of the discipline are formalized can be served as a knowledge base for supporting curriculum review, development and progress. More specifically, two curriculum ontologies can be aligned and compared to performing the existing ontology matching approaches to figure out the similarity and dissimilarity of them. The result of aligning and comparing is useful as a reference for improving the current curriculum.

\section{SYllabUS ONTOLOGY DESIGN}

The syllabus is a kind of blueprint of a course to guide students. A teacher creates a syllabus for introducing his/her course, which will be taught in the upcoming semester, and helping students to register and ready the lecture. Much important information like learning objectives, outcomes, grading policy, assignments, and learning topics is described in detail using predefined formats or templates. Nevertheless, the value of the syllabus is underestimated by students. They read and reference syllabuses only for preparing the courses, which will be taken by them in next semester. If syllabuses can be represented as well-structured and machine-readable formats, the following intelligent services for supporting 
teaching and learning can be developed.

- Syllabus matching and interlinking

- Syllabus versioning and history tracking

- Syllabus recommendation

- Syllabus-based adaptive learning pathway

- Syllabus knowledge bases with semantic searching and browsing

We design a syllabus ontology after performing the analysis of textual syllabus formats, which are available on the web, and defining classes, properties, and semantic relationships to describe a unified format. Table II shows a partial set of defined classes in the syllabus ontology. The top most abstract class is Syllabus, of which subclasses are extracted and defined from the existing formats after inventing a standard structure. Fig. 3 shows a part of the class hierarchy rooted with Syllabus class and connected with part of relations.

TABLE II: THE Classes OF SyLlaBUS ONTOLOGY

\begin{tabular}{lllll}
\hline \hline Class & Label & Superclas & SubclassOf & Definition \\
\hline Syllabus & Course Syllabus & Course & Instructor, ... & Core concept \\
Instructor & Instructor & Syllabus & Teacher, Assistant & Course instructors \\
LearningObjective & Learning Objective & Syllabus & - & Description of learning objectives \\
LearningMaterial & Learning Material and Object & Syllabus & TextBook, Article, ... & Learning materials including textbooks \\
TeachingMethod & Types of Teaching Method & Syllabus & Lecture, ... & Different kinds of teaching methods \\
LearningMethod & Types of Learning Method & Syllabus & Reading, Writing, ... & Different kinds of learning methods \\
Assignment & Assignment & Syllabus & - & Assignments \\
ExamQuiz & Exam and Quiz & Syllabus & - & Exams and quizs \\
\hline \hline
\end{tabular}

To measure the achievement of students, we formalize and design the structured format of learning objectives. Most of all learning objectives in the current syllabuses are described as textual format. For example, learning objectives, a course syllabus titled "Understanding Java Programming" may be described as follows:

- Understand the syntax and semantic of Java programming language

- Design a class through defining properties and methods

- Implement programs in the object-oriented perspective

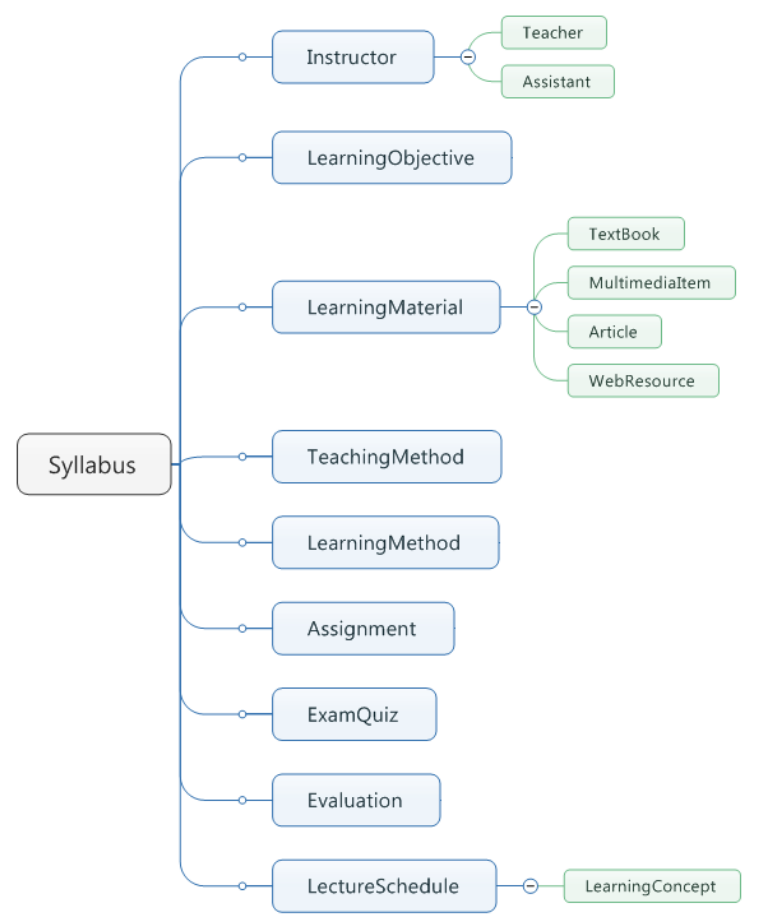

Fig. 3. The class hierarchy of the curriculum ontology.

Teachers and students read and understand textual learning objectives. However, the textual description should be formalized to perform machine-readable processing and achievement calculation automatically. We design a learning objective class and relationships depicted in Fig. 4. As you see in Fig. 4 learning objectives have connection with assignments, exams including quizzes, and learning subjects.

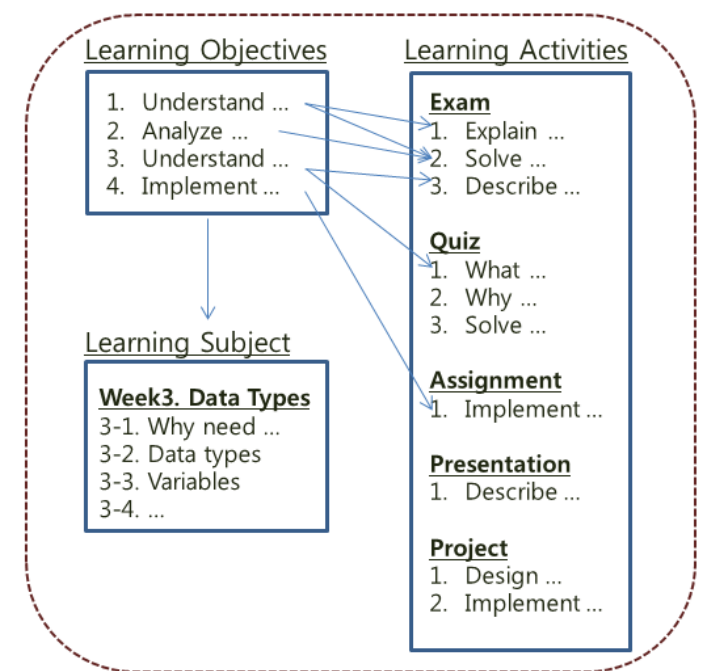

Fig. 4. Learning objectives should be related to learning subjects and activities to measure the learning achievement of students.

First of all, we reference the Boom's taxonomy represented in Table II and define the structure of learning objective class as the following tuple which is composed of five elements, identifier, learning objective, cognitive level, attitude level, and skill level.

$$
<I D, o b j_{i}, C_{j}, A_{k}, S_{p}>
$$

obj : each sentence of learning objectives

$C:$ a specific cognitive level related to obj $j_{i}$

$A:$ a specific attitude level related to $o b j_{i}$

$S:$ a specific skill level related to obj $j_{i}$

To create interlinks between learning objectives and assignments, we add identifiers of learning objectives to the related each of the assignments. For exams and learning topics, we did the same process to connect with learning objectives. In addition, we create an achievement matrix in order to measure the learning achievements of students for 
weekly learned topics as well as the entirety of a course as depicted in Fig. 5. In the achievement matrix, the columns are learning objectives and rows are students. Each cell has a value representing the degree of achievement of a certain student against a specific learning objective (see Table III).

TABLE III: THE BLOOM's TAXONOMY

\begin{tabular}{llll}
\hline \hline Levels & Cognitive & Attitude & Skill \\
\hline 1 & Knowledge & Receiving & Imitation \\
2 & Comprehension & Responding & Manipulation \\
3 & Application & Valuing & Precision \\
4 & Analysis & Organizing & Articulation \\
5 & Synthesis & Characterizing & Naturalization \\
6 & Evaluation & Cooperating & Representation \\
\hline \hline
\end{tabular}

\section{Achievement Assessment Matrix}

\begin{tabular}{|c|c|c|c|c|c|}
\hline & $\mathrm{LO}_{1}$ & $\mathrm{LO}_{2}$ & $\mathrm{LO}_{3}$ & $\mathrm{LO}_{4}$ & \\
\hline $\mathrm{s}_{1}$ & $a_{11}$ & $a_{12}$ & $a_{13}$ & $a_{14}$ & . \\
\hline $\mathrm{s}_{2}$ & $a_{21}$ & $a_{22}$ & $a_{23}$ & $a_{24}$ & ... \\
\hline$s_{3}$ & $a_{31}$ & $a_{32}$ & $a_{33}$ & $a_{34}$ & ... \\
\hline
\end{tabular}

Fig. 5. The achievement assessment matrix.

From the achievement matrix, we can figure out students who have a lower achievement for each learning objective than a specific threshold which means a minimum requirement. These unfulfilled students can be guided to study the unachieved topics by the generation of adaptive learning paths. As shown in Fig. 6 the adaptive learning paths can be generated in different granularity. The high-level learning path can be created using syllabuses and their relationships. The low-level learning path can be created using learning concepts and their relationships defined in the subject ontology.

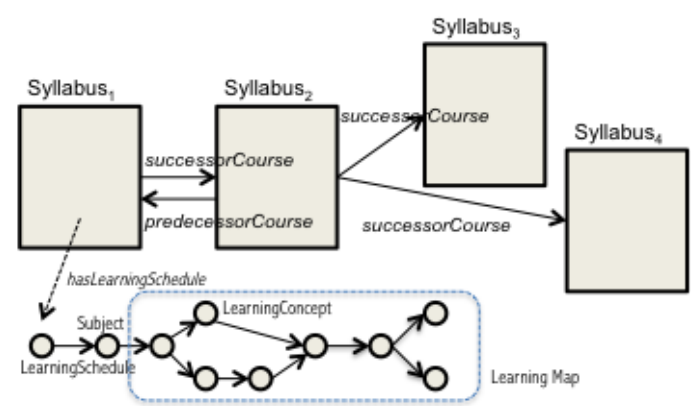

Fig. 6. Relationships created between syllabuses.

Table IV represents the semantic relationships that can be established between syllabuses. A course may have several syllabuses, but the active syllabus is only one created recently. The others are maintained with a relationship, isOldVersionOf/hasOldVersion, in the syllabus repository. Thus, we can trace the change history of a syllabus and make the syllabus to be useful with the plenty of learning-related information.

TABLE IV: RELATIONSHIPS OF SYLLABUS CLASS

\begin{tabular}{lllll}
\hline \hline \multirow{2}{*}{ Relationship } & Range & Domain & $\begin{array}{l}\text { Cardinalit } \\
\text { y }\end{array}$ & Definition \\
\hline hasOldVersion & Syllabus & Syllabus & $1: 1$ & Versioning relationship between syllabuses \\
predecessorCourse & Syllabus & Syllabus & $1: n$ & Predecessor relationship \\
successorCourse & Syllabus & Syllabus & $1: n$ & Successor relationship \\
sameCourse & Syllabus & Syllabus & $1: n$ & Equal relationship \\
relatedCourse & Syllabus & Syllabus & $1: n$ & Both of syllabuses are related \\
hasLearningMaterial & Syllabus & LearningMaterial & $1: n$ & Relathionship to materials \\
hasLearningObjective & Syllabus & LearningObjective & $1: n$ & Relationship to objectives \\
hasInstructor & Syllabus & Instructor & $1: 1$ & Relationship to an instructor \\
\hline \hline
\end{tabular}

\section{Syllabus Classification SCHEME}

\section{A. Mapping Rule Definition}

To integrate heterogeneous structured syllabuses based on a unified structured format we first define mapping rules to be used for connecting entities of different formats of syllabuses. A mapping rule denotes a tuple of a standard heading term and a set of corresponding but different terms as follows (1).

$$
\left(t_{s}, t_{1}, t_{2}, t_{3}, \ldots, t_{n}\right)
$$

In (1), $t_{s}$ denotes a standard term and the other terms, $\mathrm{t}_{\mathrm{i}}$, denote the corresponding terms. When students enter query keywords to search syllabuses, the query keywords can be expanded using mapping rules. So, a lot of relevant syllabus can be delivered to users to be considered.

\section{B. Classification Method Definition}

Syllabus classification is the organization of heterogeneous syllabuses created by different instructors based on a standard taxonomy of knowledge area. The CS2013 defines the body of knowledge as a hierarchical structure. Fig. 7 shows a partial hierarchy of Programming Language knowledge area. PL knowledge area connects 17 knowledge units directly. These knowledge units correspond to courses in the computing curriculum. Each knowledge unit has children, which denote the subjects to be learned in courses, in 2 or 3 levels.

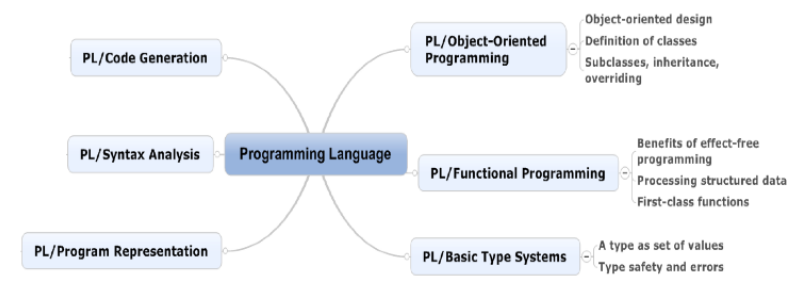

Fig. 7. Standard taxonomy of the area of programming courses.

Our syllabus classification method consists of the following steps: First, we create a knowledge area mapping table, which stores knowledge units and their corresponding 
course names. Our mapping table is defined as following expression (2).

$$
\operatorname{KMT}\left(r_{i}\right)=\left\{K_{i}, C_{i j}, W_{i}\right\}
$$

Second, morphological analysis performs to identify noun terms from the title and description segments of a syllabus. Third, the extracted terms as a result of morphological analysis can be compared to index terms of the mapping table in order to compute the string similarity between them using expression (3) and (4).

$$
\begin{gathered}
s\left(w_{i}, w_{j}\right)=\left\{\begin{array}{l}
1, w_{i}=w_{j} \\
0, w_{i} \neq w_{j}
\end{array}\right. \\
S(N, M)=\frac{\sum_{i=1}^{m} s_{i}}{|N|+|M|}
\end{gathered}
$$

Finally, the well-matched knowledge units having the similarity values higher than a predefined threshold can be identified as the ranked list. Then, a given syllabus can be classified to the best-matched knowledge unit semi-automatically.

\section{CONClusion}

In the education fields, both of curriculum and syllabus are very important for teachers and students. They can understand what they teach and learn for each semester from curricula and syllabuses. The goal of our research is the improvement of the usability of curriculum and syllabus through the design and implementation of learning ontologies. We designed the semantic model of curriculum with the purpose of organizing knowledge areas and subjects for each discipline rather than laying out courses. Curriculum ontology can be used to integrate different curricula of departments of same discipline or enable the convergence of multiple disciplines.

Our focus on design of syllabus ontology is the development of the standard knowledge structure to be used for creating syllabus repository. We created the schema of syllabus ontology to include multiple subject ontologies which conceptualize learning concepts be taught in lectures. In this paper, we present a syllabus classification scheme as well as learning ontologies. This scheme can be used to transform unstructured syllabus into an instance of syllabus ontology and then store the instance into a syllabus repository.

Our future work is the development of an intelligent service framework based on the integrated learning ontology to support curriculum design process, syllabus reference service, personal learning services, and so on.

\section{REFERENCES}

[1] N. Rubens, D. Kaplan, and T. Okamoto, "E-learning 3.0: Anyone, anywhere, anytime, and AI," in Proc. International Workshop on Social and Personal Computing for Web-Supported Learning Communities, 2011, pp. 1-11.

[2] S. Wheeler, "E-learning 3.0: Learning through the extended smart web," presented at National IT Training Conference, 2011.

[3] Y. Chi, "Developing curriculum sequencing for managing multiple texts in e-learning system," in Proc. International Conference on Engineering Education, 2010.

[4] P. Libbrecht, "Cross curriculum search through the geoskills' ontology," in Proc. SEAM'08, 2008, pp. 38-50.

[5] R. Shackelford, A. McGettrick, R. Sloan, H. Topi, G. Davies, R. Kamali, J. Cross, J. Impagliazzo, R. LeBlanc, and B. Lunt, "Computing curricula 2005: The overview report," ACM SIGCSE Bulletin, vol. 38, no. 1, 2006.

[6] W. P. F. Apple and H. S. I. Horace, "Educational ontologies construction for personalized learning on the web," Studies in Computation Intelligence, vol. 62, pp. 47-82, 2007.

[7] R. Mizoguchi, "Tutorial on ontological engineering-part 2: Ontology development, tools and languages," New Generation Computing, OhmSha\&Springer, vol. 22, no. 1, 2004.

[8] R. Mizoguguchi and J. Bourdeau, "Using ontological engineering to overcome AI-ED problems," International Journal of Artificial Intelligence in Education, vol. 11, no. 2, pp. 107-121, 2000.

[9] R. Marco and S. Joseph, "Curriculum management and review - An ontology-based solution," Technical Report \# DIT-07-021, University of Trento, 2007.

[10] M. Nilsson, M. Palmer, and J. Brase, "The LOM RDF binding Principles and implementation," in Proc. The 3rd Annual ARIDNE Conference, Lueven, Belgium, 2003.

[11] D. G. Sampson, M. D. Lytra, G. Wagner, and P. Diaz, "Guest editorial: Ontologies and the semantic web for e-learning," Educational Technology and Society, vol. 7, no. 4, pp. 26-28, 2004.

[12] X. Yu, M. Tungare, W. Fan, M. Perez-Quinones, and E. Fox, "Using automatic metadata extraction to build a structured syllabus repository," in Proc. the 10th Interna-tional Conference on Asian Digital Libraries, 2007.

[13] M. Sahami, M. Guzdial, A. McGettrick, and S. Roach, "Setting the stage for computing curricula 2013: Computer science - Report from the ACM/IEEE-CS joint task force," in Proc. SIGCSE11, 2011.

[14] ACM/IEEE-CS Joint Task Force on Computing Curricula, Computer Science Curricula 2013, ACM Press and IEEE Computer Society Press, 2013.

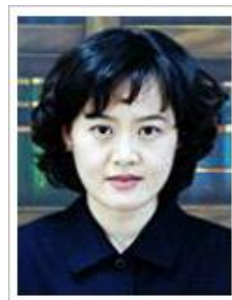

Hyunsook Chung is a professor in the Department of Computer Engineering at Chosun University, Gwang-Ju, Korea. She received her B.S. degree in physics in Daegu Catholic University and a M.S. degree in computer engineering from same university. She received a Ph.D. degree in computer engineering from Yonsei University in 2003. Her research interests include multimedia data processing, knowledge data processing, ontology engineering, and information processing.

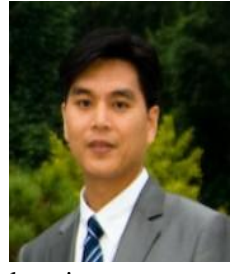

learning.
Jeongmin Kim is a professor in the Department of Computer Engineering at Daejin University, Pocheon, Korea. He received his B.S. degree and a M.S. degree in computer science in Hongik University in 1992 and 1994 respectively. He received his Ph.D. degree in computer engineering from Seoul National University in 2007. His research interests include semantic web, semantic information processing, IPTV, and smart 